\title{
MICRO-CONTROLLER BASED LI-FI ENABLED LOCALIZED INFORMATION ROUTER
}

\author{
Rishabh Raghav, Saurabh Kumar Yadav, Rohit Pandey, Ajay Pratap Singh, Shubham Tiwari \\ E-Mail Id: rishabh.16ben1018@abes.ac.in, saurabh.16ben1061@abes.ac.in \\ ABES Engineering College, Ghaziabad, Uttar Pradesh, India
}

\begin{abstract}
Li-fi which is also referred to as Visible Light Communication (VLC), is a combination of "Wi-Fi" and "Light". As we all know that the conventional Wi-Fi uses radio waves for transmitting and receiving data but in our project visible light will be used for the same. As a state-of-the-art technology, it's application can be found everywhere in the world, both in the field of industry as well as research; mostly because of its economic viability, high expandability and extreme efficiency and performance. Our project, although introductory, may be an extensive validity of concepts, with enhanced circuits and an extended communication truce executed simultaneously to successfully implement the sending and receiving of various data or information.
\end{abstract}

Keywords: Li-Fi (Light Fidelity), Wi-Fi (Wireless Fidelity), VLC (Visible Light Communication), LOS (Line of Sight).

\section{INTRODUCTION}

We are living in a world where data communication has become very important and integral part of every one life. We are very much dependent on internet for our day to day activities and due to this the speed of internet is becoming slow with respect to the need of humans. Till this point of time we are generally using Wi-Fi for the transmission of data and information which provide maximum speed up to $150 \mathrm{Mbps}$. But due to the need for sending and receiving huge amount of data the speed is not feasible. As the traffic increases i.e. no of connections increases the speed of Wi-Fi decreases.

In order to overcome this, we are moving towards a new technology which is known to be Li-Fi. Li-Fi basically uses visible light in order to establish communication. It is also known as VLC (Visible Light Communication). It is also a modern and effective way of wireless communication. In this we use LED's for the transmission and receiving the data and it proves to be a very efficient way for both communication as well as for lighting purpose. The signal which is sent over a range by changing the light intensity at high speed so that it cannot be detected by the human eyes. With the help of this we can achieve speed up to several GBPS. and the data transmission is also considered to be secure and un-hackable unlike Wi-Fi and others.

\section{OBJECTIVE OF THE PROJECT}

The main objective of the project is to achieve data transfer and communication with the help of visible light. We will be developing a small working prototype with a UI which will control the data flow with the help of several circuitry components.

\section{THEORETICAL STUDY}

\subsection{Working Principle}

The working principle of Li-Fi is pretty simple and it is based on concept visible light communication. Li-Fi uses the visible light spectrum which ranges from $400 \mathrm{THz}$ to $800 \mathrm{THz}$. In this we use many LED lights interconnected with each other in order to forma a wireless communication network. In our conventional wi-fi systems there are possibility that our data can get off track deface curtsey of the radio wave interference but it does not happen as with $\mathrm{Li}-\mathrm{Fi}$. The concept behind the working of $\mathrm{Li}-\mathrm{Fi}$ is that whenever electric current is given to the interconnected lights then it releases large number of sunshine. Now the intensity of the light bulb is varied in a very fast way so that it is not seen by the normal human eye. With the change of the intensity of light bulb the analog or raw data is converted into binary form i.e. in the form of 0's and 1's. Now this binary signal is sent with the help of a transmitter from one area to another. At the receiver end there is another circuit which is called receiver whose work is to receive the transmitted signal. Now after the signal is received its strength is modulated with the help of some hardware circuit component and then again it is converted back to its original from and displayed on the screen. This communication is very fast and also efficient.

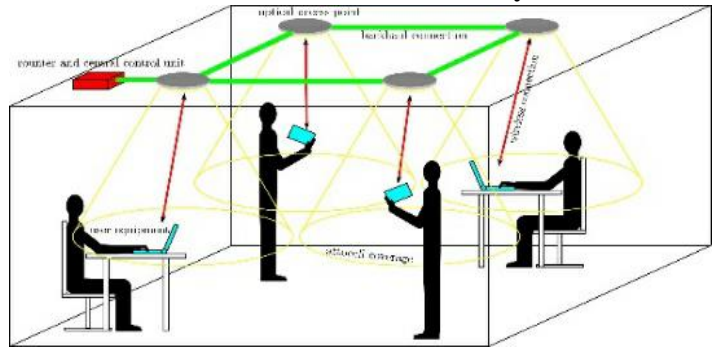

Fig. 3.1 LI-Fi Applied to Indoor Wireless Network [5]

DOI Number: https://doi.org/10.30780/specialissue-ICACCG2020/004 
The working prototype will consist of mainly two sections that are transmitter and receiver sections. Several steps associated with the transmission of information are as follow:

$>$ Changing of raw data into its binary format.

$>$ For more effective data transfer, executing effective networking concord.

$>$ Making sure that data is transferred at high speed with the help of suitable circuit components. The various steps involved in receiving of the data are:

$>$ Making sure that proper data is received with the help of suitable circuit components.

$>$ For the next steps, enhancing the received data signals.

$>$ Removing the unwanted noise signals with the help of proper hardware elements in order to have a more efficient and fast data processing.

$>$ Analog to digital signal conversion with the help of ADC.

$>$ Taking the useful and original data from the data received and showing the information.

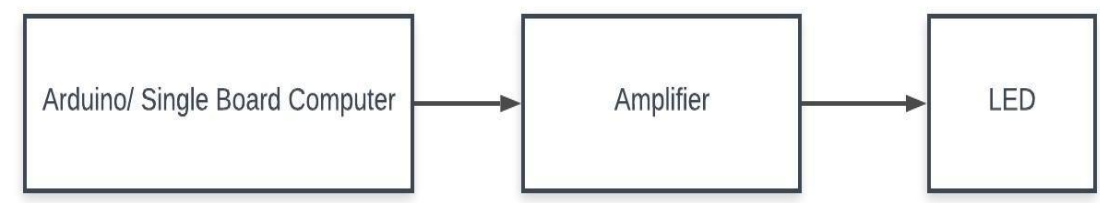

Fig. 3.2 Transmitter Section

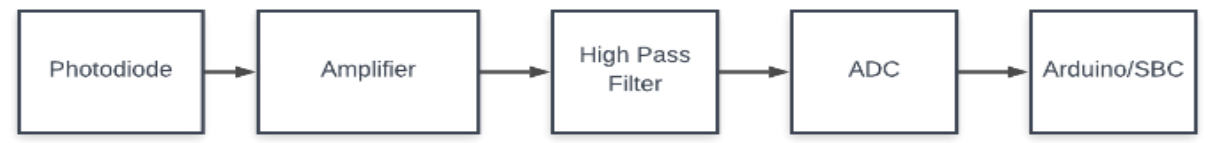

Fig.3.3 Receiver Section

\subsection{Technical Requirement}

\subsubsection{Hardware at Transmitter End}

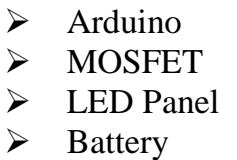

\subsubsection{Hardware at Receiver End}

$>$ Photodiode

$>$ Amplifier

$>$ High Pass Filter

$>$ Analog to Digital Converter

$>$ Arduino

\subsubsection{Software Used}

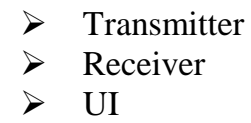

\subsection{Block Diagram}

The block diagram of this consist of mainly two section one is receiver and other is transmitter. The block diagram is given below:

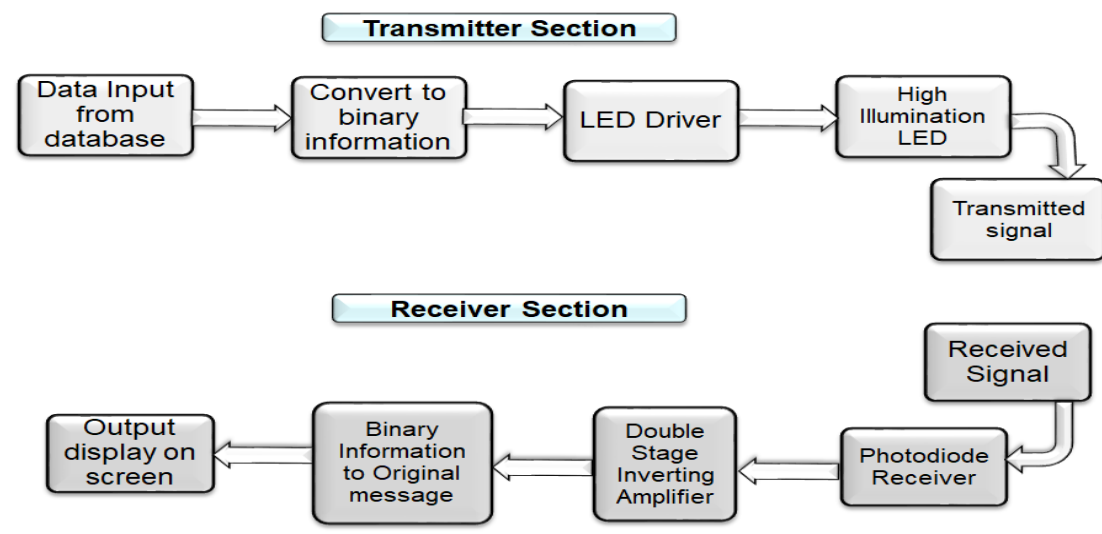

Fig. 3.4 Block Diagram of Data Flow with the help of Li-Fi

DOI Number: https://doi.org/10.30780/specialissue-ICACCG2020/004 
ICACCG2020 30-31 July, 2020, Ansal University, Gurgaon, India

International Journal of Technical Research \& Science (Special Issue)

ISSN No.:2454-2024 (online)

\section{ADVANTAGES AND DISADVANTAGES}

The microcontroller based li-fi router has many advantages over normal router which are given below:

$>$ The transmission of the information with the help of li-fi is very fast. It can speed up to some GBPS.

$>$ One of the major advantages of li-fi is that it can be used in the areas which are sensitive to radio frequency like airplanes, hospitals etc.

$>$ It can also be seen that with the use of LED, the transmission process become more energy efficient because the LED's are very much energy efficient.

$>$ It can also be observed that the cost of maintenance is very much low as compared to conventional ones in their long run [15].

$>$ Since Li-Fi works on the principle of line of sight and due to this the data or information is secured. [17].

Disadvantages are as follows:

$>$ It does have limited range because light rays are not able to penetrate or pass through the walls, opaque objects etc [17].

$>$ The cost for the initial installation of this technology is considered to be on the higher note [19].

\section{HARDWARE AND COST ANALYSIS}

The hardware is shown as Fig.5.1.

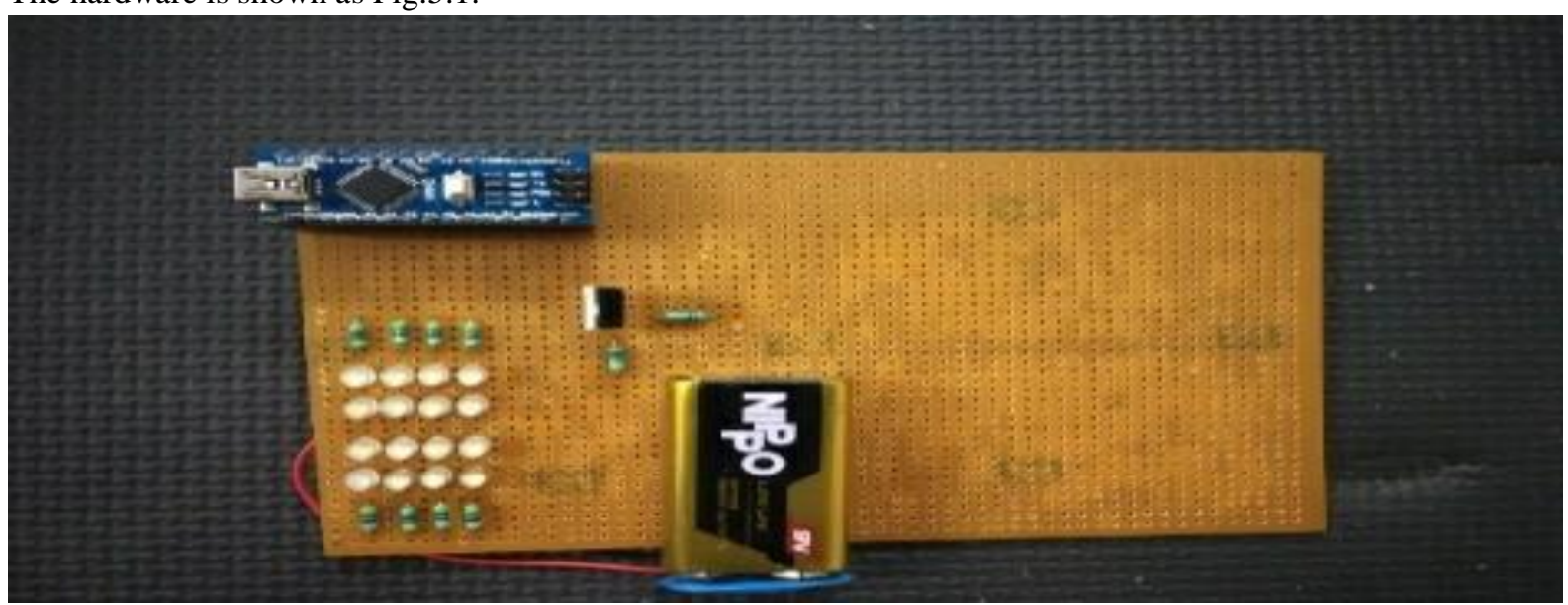

Fig. 5.1 Proposed Hardware Part of Transmitter Section

Table-5.1 Estimated Cost Analysis of the Project

\begin{tabular}{|l|l|l|l|}
\hline S. No. & Description & Qty. & Price (Rs) \\
\hline 1 & LED Panel & 2 & 200 \\
\hline 2 & BJT & - & 20 \\
\hline 3 & Resistors & - & - \\
\hline 4 & Arduino Microcontroller & 2 & 2000 \\
\hline 5 & Power Supply & 2 & 40 \\
\hline 6 & Photodiode & - & - \\
\hline 7 & Comparators & - & 180 \\
\hline 8 & TTL Converter & - & 220 \\
\hline 9 & Bluetooth Module & 2 & 1000 \\
\hline TOTAL & & & 3660 \\
\hline
\end{tabular}

\section{RESULTS AND FINDINGS:}

\subsection{Hardware Development}

Since this topic i.e. the li-fi is a growing area in which a huge amount of research in going on, we took help of few papers and some project which helped us a lot. At the starting phase we implemented many process, elements and circuitry designs.

\subsection{Speed and Range}

With the help of our hardware design and program we are able to transmit data at a speed of $2 \mathrm{kbps}$ and that to up to distance of $1 \mathrm{ft}$. 


\section{FUTURE PROSPECT}

\subsection{The Dual Advantage}

It will surely be an added advantage to our upcoming generation in the future because it will give us both the services of lighting as well as internet communication.

\subsection{Alternative of Wi-Fi}

In the upcoming future this will surely replace the wi-fi systems completely. It has more advantages over the latter. It can be used even at places where the wi-fi causes interference problems like hospitals, airplanes etc. Also, it will help in the transfer of huge amount of data.

\subsection{Secure}

The line of sight feature in li-fi is an advantage as well as a disadvantage. If we go out from LOS the data transfer will not be done but it also provides added advantage of security by which our data is safe inside a closed area.

\section{CONCLUSION}

Li-Fi technology has power to connect secluded and obscure terrains across the world that can't be outreached with the help of glass fibre. It's very much utilized in smart-city projects. The use of Li-Fi is increasing and growing rapidly because of increased and rapid development of several smart cities across the world. Li-Fi technology thus holds the answer to varied short comings of radio frequency based wireless data communication systems. It's a good range of applications. It also supports green and pollution free environment, because in this visible light communication is used for transmission of knowledge and data which is harmless and available everywhere. In the upcoming future it can be useful in the several areas of development like bigdata, AI, ML etc. Moreover, while there are 300 gigahertz of RF spectrum and there's 300 terahertz of light spectrum. If you add spectrum of infrared with that of the light spectrum, it'll be around two thousand six hundred (2600) times larger than the whole radio-frequency spectrum. The chances are vast and may be investigated in the future. If this state-of-art is often applied into real use, every bulb can be frequently used as a wireless transmitter like Wi-Fi hotspot in order to share data and transmit information and that the world will proceed on the way to an Immaculate, Greener, Secure and Scintillating Future.

\section{REFERENCES}

[1] H. Haas, Wireless data from every light bulb, TED Global, August 2011.

[2] H. Haas, L. Yin, Y. Wang, C. Chen, What is LiFi? IEEE J. Light. Technol. 34 (6) (2016) 1533-1544.

[3] P.J. Winzer, D.T. Neilson, From scaling disparities to integrated parallelism: a decathlon for a decade, IEEE J. Light. Technol. 35 (5) (2017) 1099-1115.

[4] F. Boccardi, R.W. Heath, A. Lozano, T.L. Marzetta, P. Popovski, Five disruptive technology directions for 5G, IEEE Commun. Mag. 52 (2) (2014) 74-80.

[5] M.S. Islim, et al., Towards $10 \mathrm{~Gb} / \mathrm{s}$ orthogonal frequency division multiplexing-based visible light communication using a GaN violet micro-LED, Photon. Res. 5 (2) (2017) A35-A43.

[6] D. Tsonev, S. Videv, H. Haas, Towards a $100 \mathrm{~Gb} / \mathrm{s}$ visible light wireless access network, Opt. Express 23 (2) (2015) $1627-1637$.

[7] Stefan, H. Burchardt, H. Haas, Area spectral efficiency performance comparison between VLC and RF femtocell networks, IEEE International Conference on Communications (ICC), 2013, pp. 3825-3829.

[8] Y. Liang, H. Haas, Physical-layer security in multiuser visible light communication networks, IEEE J. Sel. Areas Commun. (2017).

[9] W. Ni, R.P. Liu, B. Collings, X. Wang, Indoor cooperative small cells over ethernet, IEEE Commun. Mag. 51 (9) (2013) 100-107.

[10] Papaioannou, F.N. Pavlidou, Evaluation of power line communication equipment in home networks, IEEE Syst. J. 3 (3) (2009) 288-294.

[11] H. Haas, High-speed wireless networking using visible light, SPIE Newsroom (2013).

[12] V.H. MacDonald, The cellular concept, Bell Syst. Tech. J. 58 (1) (1979) 15-43.

[13] Goldsmith, Wireless Communications, Cambridge University Press, 2005.

[14] D. Tsonev, S. Videv, H. Haas, Unlocking spectral efficiency in intensity modulation and direct detection systems, IEEE J. Sel. Areas Commun. (99) (2015) 1.

[15] H. Elgala, T.D.C. Little, SEE-OFDM: Spectral and energy efficient OFDM for optical IM/DD systems, IEEE 25th Annual International Symposium on Personal, Indoor, and Mobile Radio Communication (PIMRC), 2014 2014, pp. 851-855.

[16] H. Ma, L. Lampe, S. Hranilovic, Robust MMSE linear precoding for visible light communication broadcasting systems, 2013 IEEE Globecom Workshops (GC Wkshps), 2013, pp. 1081-1086.

[17] Tzanakaki, et al., Wireless-optical network convergence: enabling the 5G architecture to support operational and enduser services, IEEE Commun. Mag. 55 (10) (2017) 184-192.

[18] Ghimire, H. Haas, Self-organising interference coordination in optical wireless networks, EURASIP J. Wirel. Commun. Netw. 1 (131) (2012).

[19] Chen, V.S.,D. Tsonev, H. Haas, Fractional frequency reuse in DCO-OFDM-based optical attocell networks, J. Light. Technol. 33 (19) (2015) 3986-4000.

[20] Y. Wang, S. Videv, H. Haas, Dynamic load balancing with handover in hybrid Li-Fi and Wi-Fi networks, Proc. IEEE 25th International Symposium on Personal Indoor and Mobile Radio Communications (PIMRC), 2014, pp. 548-552. 